\title{
Mycobacterium Sputum Analysis and Comparative Evaluation in Pulmonary Tuberculosis Laboratory Diagnosis in Ethiopia
}

\author{
Desta $\mathrm{KT}^{1 *}$, Dolamo $\mathrm{BL}^{2}$ and Endris $\mathrm{IH}^{3}$ \\ 1 University of South Africa, Addis Ababa regional learning center, South Africa \\ 2University of South Africa, South Africa, Pretoria, South Africa \\ ${ }^{3}$ Federal Ministry of Health of Ethiopia, Addis Ababa, Ethiopia
}

\section{Research Article \\ Volume 2 Issue 9}

Received Date: August 30, 2018

Published Date: September 07, 2018

*Corresponding author: Kassaye Tekie Desta, University of South Africa, Addis Ababa regional learning center, South Africa, Email: kassayetek@gmail.com

\section{Abstract}

Background: Even though the laboratory testing is essential for the diagnosis and control of tuberculosis (TB), it does not receive enough attention in Ethiopia where acid-fast bacilli (AFB) sputum microscopy is often the only available method to diagnose TB. The low detection rate of Mycobacterium tuberculosis in Ethiopia using sputum microscopy and the limitation of chest-x-ray for mass screening and diagnosis initiated the researchers to conduct the study.

Objective: The objective of this study was to make a comparative analysis of chest $\mathrm{x}$ - ray, sputum microscopy and culture for diagnosis of Mycobacterium tuberculosis.

Methods: Quantitative, retrospective cross-sectional study was conducted to analyses chest x-ray, sputum microscopy and culture for diagnosis of Mycobacterium tuberculosis. The study was conducted on data collected from International Clinical Laboratories (ICL) in Ethiopia. Samples were selected from the logbooks using systemic random sampling and until the required sample number of 943 had been obtained. Data collection was done using standardized checklist from 943 participants' documents from February 2010 to March 2011. The 943 study participants' documents sampled were analyzed for x-ray, AFB microscopy, solid culture, liquid culture and anti-TB drug susceptibility testing.

Results: Among the 943 study participants' documents with suggestive chest x-ray findings; $13(1.4 \%)$ had a positive smear results for AFB. The total number of organisms recovered was 98 of which 23 were M. tuberculosis whereas 75 were non-tuberculosis mycobacterium (NTM). In this study significant difference was not demonstrated statically between BACTEC Mycobacterium growth indicator tube (MGIT) 960 liquid medium and Lowenstein Jensen (LJ) solid medium in terms of Mycobacterial recovery rate ( $p>0.05)$.

The diagnostic value of AFB sputum microscopy against sputum culture method for diagnosis of tuberculosis in terms of sensitivity, specificity, and positive and negative predictive values of serial AFB screening among these groups were $26 \%$, $99.2 \%, 46 \%$, and $98.2 \%$ respectively. The resistance patter to isoniazid; rifampin, ethambutol and streptomycin was $30.5 \%$ ), $8 \%, 26 \%$ and $22 \%$ respectively. $2.1 \%$ participants' documents were found to be multidrug resistant (MDR). 


\section{Virology \& Immunology Journal}

Conclusion: The findings of this study revealed that chest x-ray for TB screening lacks significant specificity and is susceptible to miss screening and diagnosis. The findings of this study also showed that culture method has a great potential to improve the clinicians' ability to diagnose TB.

Keywords: TB; Chest X-Ray; Culture; MDR; Sputum Microscopy; AFB; TB-Registers

\section{Introduction}

Mycobacterium tuberculosis and the regional variants or subtypes Mycobacterium africanum and Mycobacterium canetti are primarily pathogenic in humans. Tuberculosis is most commonly caused by M. tuberculosis [1]. TB affects all age groups but the risk of developing the disease increases considerably when TB infection co-exists with an alteration in the immune system; such as co-infection with human immune deficiency virus [2].

Diagnosis of suspected TB cases rapidly and accurately is crucial for the global strategies of tuberculosis (TB) control. Despite the significant progress made in TB diagnostics of developed countries, in developing countries, the techniques used for diagnosing TB have remained relatively unchanged for many years [3]. The old TB diagnostic tools are unreliable, time consuming compared to the new TB tools which are easy to run and high sensitivity. Lack of access to good diagnostic tools in developing and high burden countries at all health service levels leaves many patients undiagnosed [4].

Ethiopia ranks seventh among the world's 22 highburden countries severely affected by $\mathrm{TB}$, as recognized by World Health Organization (WHO) in its tuberculosis global report [5]. According to the report, the estimated incidence of all forms of tuberculosis was $314,000 / 100000$ population per year and smear positive was $163,000 / 100000$ population. Tuberculosis mortality rate was 92,000/100000 and the prevalence of HIV positivity in tuberculosis incidence cases of all ages was reported as $19 \%$. The 22 high TB burden countries are: Afghanistan, Bangladesh, Brazil, Cambodia, China, Congo, Ethiopia, India, Indonesia, Kenya, Mozambique, Myanmar, Nigeria, Pakistan, Philippines, Russian Federation, South Africa, United Republic of Tanzania, Thailand, Uganda, Viet Nam and Zimbabwe [3,5].

TB cases in foreign-born persons account for more than $50 \%$ of all TB cases in the United States [6]. Furthermore, tuberculosis rates among foreign-born person are 8 times higher than those among US-born person [7]. Even though there are no studies done on oversea screening in Africa, in terms of global distribution of the burden of TB, in 2003 the South Asia region notified $35 \%$ of all cases, the Africa region $24 \%$, and the Western region $22 \%$. The 22 high -burden countries account for approximately $80 \%$ of all estimated new TB cases each year.

This study was conducted in light of comparing chest $\mathrm{X}$-ray and sputum microscopy with culture, the gold standard for the diagnosis of $\mathrm{M}$. tuberculosis which is implemented in ICL in Ethiopia [8]. The ICL further conduct drug susceptibility test for M. tuberculosis. This study was also extended to drug susceptibility test to show the importance of this area of screening on TB diagnosis. Antimicrobial susceptibility testing is critical in prescribing an effective drug regime for a TB patient, especially in areas where drug resistance incidence is high. It is also important in the follow-up of patients who are on antimicrobial therapy but are not responding to therapy. Drug susceptibility testing is an integral part of the WHO Directly Observed Therapy Short term (DOTS)plus program [9].

The incidence of TB in developing countries is higher than in industrialized countries. Migrants from developing countries have a higher risk of being carriers of active TB than the population of the regions they enter [10]. In some countries this risk is considered serious enough to justify screening measures, which may be performed before entry, at the border, or after entry [11].

Overseas screening of TB has been implemented by United States of America (USA) for immigrants and refugees from high tuberculosis burden countries i.e. countries with WHO estimated TB incidence rate greater than 20 cases per 100,000 populations applying to reside permanently in the US [12]. The 22 high burden countries are among these in this category. In Ethiopia this screening is performed by health facilities selected by American Embassy in Ethiopia and IOM.

Sputum microscopy lacks sensitivity, especially in children and in people living with HIV/AIDS. They don't 


\section{Virology \& Immunology Journal}

produce enough sputum and become smear negative TB. In smear negative TB cases and even smear positive cases, radiographic changes are helpful in disease diagnosis. Although some chest X-ray findings are not specific for pulmonary tuberculosis, clinical manifestations and epidemiologic findings are very diagnostic [13]. Chest Xray detects the presence of lesions suggestive of TB in short time but it is highly dependent on individual performance as the method is subject to the experience of the reader and is susceptible for over- and underreporting [14]. Improving the tuberculosis laboratory diagnosis to culture technique is very crucial. Assessing the performance of the new TB screening method employed in ICL in Ethiopia is an area that has to be studied as it is a combination of chest x-ray findings, AFB microscopy and culture methods. This will significantly contribute to the low tuberculosis detection rate in Ethiopia if it is adopted nationally. Comparison of the conventional tuberculosis diagnostic techniques is very crucial in tuberculosis prevention and control in Ethiopia.

In low-resource countries, like Ethiopia, the only methods available for confirming TB in patients with a clinical presumption of active disease is AFB smear microscopy. It is rapid, inexpensive and is a very useful method to identify highly contagious patients. Despite this it has low detection capacity in detecting different stages of TB. The TB detection rate of AFB microscopy in Ethiopia is from $15-31 \%$ which is significantly lower than the $80 \%$ target set by WHO TB global report [5].

Early identification and treatment of TB in immigrants is essential because delays can result in more severe disease for the patient and disease transmission in both host and receiving countries. However, data suggest that some TB cases in foreign-born persons could be identified earlier by enhancing overseas screening [6]. Before 2007, USA overseas TB screening was based on AFB smears and $\mathrm{X}$-ray findings only. As a result, person with AFB smearnegative, $M$. tuberculosis culture-positive pulmonary TB would be missed. Such persons can be infectious. It has been estimated that AFB smear-negative pulmonary TB is responsible for approximately $17 \%$ of transmission in the US [15].

Culture is used to detect cases with low Mycobacterium loads and is also requested in cases at risk of drug-resistant tuberculosis for drug susceptibility testing, or in cases where disease due to another member of the Mycobacterium genus is suspected. AFB smear microscopy and culture can also be used for treatment effectiveness monitoring and patient follow up $[8,16]$.
AFB smear microscopy provides a preliminary diagnosis of mycobacterial disease, while the isolation of mycobacteria on culture provides a definite diagnosis of $\mathrm{TB}$ or disease due to mycobacteria other than $\mathrm{M}$. tuberculosis or NTM. Culture technique is more sensitive and specific tool compared to AFB microscopy and play great role in the diagnosis of mycobacterial disease $[17,18]$. Patients with infectious TB may remain undetected due to unreliable laboratory diagnosis. In addition, errors in AFB smear microscopy reading can result in patients being put on treatment without having the disease. Poor quality of the sputum specimen often results in AFB smear microscopy negative results [19].

Sputum is the most common specimen received for TB diagnosis. The minimum number of bacilli needed to detect their presence in stained smears has been estimated to be 5,000-10,000 per ml of sputum. For diagnosis, the sensitivity of AFB smear staining relative to culture has been estimated to vary from $50 \%$ to over 80 $\%$ [4]. While as many as two thirds of sputum smearpositive cases probably remain undetected worldwide, the efforts to control the disease have focused more on curing TB cases than on detecting them [20]. AFB microscopy is easy and quick, but it does not confirm TB diagnosis because mycobacteria other than $M$. tuberculosis are also AFB in the smear microscopic examination. In addition, a high bacterial load is needed in the specimen to render an AFB microscopy result positive. Culture techniques have been estimated to detect as many as 10-1,000 viable Mycobacteria per ml of specimen. Therefore, although most TB control programs do not support its widespread use due to enhanced laboratory complexity, biohazard and cost, all specimens should be submitted to culture, regardless of AFB smear microscopy results. A positive culture for $\mathrm{M}$. tuberculosis confirms the diagnosis of active disease [20].

The BACTEC TB-460 system was the first semiautomated system to appear on the market for mycobacteria culturing, and still serves as the benchmark for quality, reliability and performance. In the last decade, several new commercial culture media have been introduced, such as the Mycobacteria Growth Indicator Tube (MGIT) and BACTEC MGIT960 systems. Like BACTEC TB- 460, these newer systems are based on liquid media [21]. Liquid culture media has been proven to be significantly more sensitive than egg-based solid media for the isolation of mycobacteria from clinical specimens [22]. However, one disadvantage is the much higher price, which is often too high for cost-effective TB diagnosis in resource-limited countries. The final species identification of $M$. tuberculosis is based on 
characteristics such as slow growth, colony morphology, and biochemical tests [23].

\section{Research Purpose}

The purpose of the study was to compare sputum laboratory test analysis for mycobacterium tuberculosis from the study participants' documents at international clinical laboratories in Ethiopia. This study was also extended to drug susceptibility test to show the importance of this area of screening on TB diagnosis.

\section{Research Objectives}

The objective of this study was to compare mycobacterium sputum analysis of microscopy and culture methods of TB laboratory diagnosis; generate evidence to improve the existing TB diagnosis method in Ethiopia. The study was guided by the following objectives.

- To conduct comparative analysis of chest X-ray with sputum microscopy for diagnosis of TB

- To determine the sensitivity of acid-fast microscopy with culture methods

- To compare the antimicrobial susceptibility testing result of study participants' records from the liquid culture result

\section{Methods}

Quantitative, retrospective cross-sectional study was conducted to analyses chest ray, sputum microscopy and culture for diagnosis of Mycobacterium tuberculosis. The study was conducted on data collected from International Clinical Laboratories. All prospective participants' documents who had undergone chest radiography screening in Cathedral hospital, Chico hospital, St Yared hospital and IOM and with suggestive $\mathrm{x}$-ray findings from February 2010 to March 2011 were included in the study. They were all referred to ICL from these health care facilities for AFB and culture diagnosis.

The sampling frame for this study was the TB register that had all patients with suggestive $\mathrm{x}$-ray findings referred to International clinical laboratory starting from 01/02/2010 to 01/03/2011. Samples were selected from the logbooks using systemic random sampling and participants were added until the required sample number of 943 had been obtained. Data collection was done using standardized and modified checklist from 943 participants' documents tuberculosis laboratory registers of International Clinical Laboratories from February 2010 to March 2011. Demographic and laboratory data were

\section{Virology \& Immunology Journal}

obtained from laboratory registers of International Clinical Laboratories. The data collection was done by data collectors under supervision of the principal investigator.

After all the 943 study samples had been collected using the standard check list, the researcher reviewed all the check lists for completeness and performed appropriate data cleaning. Data were entered using EXCEL and SPSS versions 17 were used for statistical analysis. Frequencies, proportions and summary statistics were used to describe the study subjects in relation to relevant variables. For comparing the results of different test procedures, sensitivity, specificity and predictive values were calculated along with their 95\% confidence interval. Descriptive statistics were used to summarize socio-demographic profiles and laboratory results. These were mean, standard deviation and range. Analytic statistics of Chi square test was calculated to determine the strength of relationships between selected variables of age, sex and infection status. A p-value $<0.05$ was considered significant [6].

\section{Ethical Considerations}

Ethical clearance certificate to conduct the study was obtained from Addis Ababa City Administration Health Bureau Ethical Clearance Committee (Reference number: 7163/227). In Ethiopian health system context, a research conducted in health areas need permission from the respective health bureau where the research is conducted. Permission to use the data on the registers was obtained from the International Clinical Laboratories, Addis Ababa, Ethiopia. This was done as soon as ethical approval was granted by the Research and Ethics Committee of the Department of Health Studies, University of South Africa.

\section{Results}

From February 2010 to March 2011, eleven thousand two hundred $(11,200)$ participants' documents were screened for tuberculosis. One thousand eight hundred eighty-six participants documents' (1886) were found with chest x-ray suggestive of tuberculosis. Out of these, nine hundred forty-three (943) odd numbered study participants' documents with chest $\mathrm{x}$ - ray suggestive of TB were selected for this study. The prevalence of patients with suggestive $\mathrm{x}$-ray findings was $17.2 \%(1886 / 11200)$.

The minimum age for the study subjects was 1 (one year) and the maximum age was 99 (ninety-nine years). The mean age was 29 and standard deviation was 16 . The 


\section{Virology \& Immunology Journal}

age range for the study subjects was 98(1-99) as shown in the Table 1. Out of the total 943 study participants' documents with suggestive chest X-ray findings, $481(51 \%)$ were male and $462(49 \%)$ were female. Three hundred nine $(32.4 \%)$ of the study participants were in the age range of 29-44, which indicated that this age group are more likely to migrate for better job and other opportunities abroad.

\section{Mycobacterium Diagnosis Result \\ Tuberculosis \\ Laboratory}

The 943 study participants' documents sampled were analysed for CXR, AFB microscopy, solid culture, liquid culture and anti-TB drug susceptibility testing. Among the 943 study participants' documents with suggestive chest x-ray findings $13(1.4 \%)$ had a positive smear results for AFB (Table 1).

\begin{tabular}{|l|c|c|}
\hline \multicolumn{1}{|c|}{ AFB and culture results } & $\begin{array}{c}\text { Nubmer of study } \\
\text { participant }\end{array}$ & $\begin{array}{c}\text { Percentage of study } \\
\text { participant }\end{array}$ \\
\hline Positive AFB sputum smear results & 13 & $1.40 \%$ \\
\hline Positive M. tuberculosis culture results & 23 & $2.40 \%$ \\
\hline Positive AFB sputum smear status and positive M.tuberculosis culture results & 6 & $0.60 \%$ \\
\hline Positive AFB sputum smear and negative M.tuberculosis culture results & 7 & $0.70 \%$ \\
\hline Negative AFB sputum smear status and positive M.tuberculosis culture results & 17 & $1.80 \%$ \\
\hline Negative AFB sputum smear status and negative M.tuberculosis culture results & 913 & $96.80 \%$ \\
\hline
\end{tabular}

Table 1: Characteristics of study participants' documents ( $n=943)$.

Characteristics of the findings revealed that twentythree ( $n=23)$ study participants' documents with suggestive chest X-ray findings were found to be positive Mycobacterial culture and identified as $\mathrm{M}$. tuberculosis. Seventeen (17) whose smears were negative for AFB found to be positive for Mycobacterial culture and identified as M. tuberculosis (Table 1). Among 943 study participants' documents with abnormal chest radiographs, $13(1.4 \%)$ had positive acid-fast bacilli smear results, which is equivalent to 120 in 100,000 population. Twenty-three $(2.4 \%)$ had positive Mycobacterium tuberculosis culture results (pulmonary TB disease) and needed anti-TB treatment. In this study, 930/943(98.6\%) of the study participants' documents with chest x-ray suggestive of TB were found to be negative for AFB.

The following cross table compares the diagnostic value of AFB sputum microscopy against sputum culture method for diagnosis of TB (Table 2).

\begin{tabular}{|c|c|c|c|}
\hline \multicolumn{5}{|c|}{ Culture result status } \\
\hline AFB result status & Positive & Negative & Total \\
\hline Positive & 6 & 7 & 13 \\
\hline Negative & 17 & 913 & 930 \\
\hline Total & 23 & 920 & 943 \\
\hline
\end{tabular}

Sensitivity $=6 / 23=26 \%$

Specificity $=913 / 920=99.2 \%$

Positive predictive value $=6 / 13=46 \%$

Negative predictive value $=913 / 930=98.2 \%$

Table 2: Sputum Culture and AFB status of the participants' documents ( $\mathrm{n}=943)$.
The sensitivity, specificity, and positive and negative predictive values of serial AFB screening among this group were $26 \%$ (6/23), 99.2\% (913/920), 46\% (6/13), and $98.2 \%(913 / 930)$, respectively (Table 2$)$.

\section{Comparison of Solid Culture with Liquid Culture for the Diagnosis of TB}

Conventional solid medium such as the egg-based LJ traditionally have been used for the recovery of Mycobacteria from clinical material however, the slow growth rate of many pathogenic Mycobacterium species on solid medium can substantially delay the identification process. Automated BACTEC MGIT 960 system have been developed to enhance the efficiency and time of detection. Table 3 below compares rate of recovery by both solid and liquid cultures.

\begin{tabular}{|c|c|c|}
\hline \multirow{2}{*}{$\begin{array}{c}\text { Culture } \\
\text { Medium }\end{array}$} & \multicolumn{2}{|c|}{ No of isolates recovered } \\
\cline { 2 - 3 } & $\begin{array}{c}\text { M. } \\
\text { tuberculosis } \\
\text { (n=23) }\end{array}$ & $\begin{array}{c}\text { Non-tuberculosis } \\
\text { Mycobacterium(NTM), } \\
\mathbf{n = 7 5}\end{array}$ \\
\hline $\begin{array}{c}\text { BACTEC MIGIT } \\
\text { 960 (Liquid) } \\
\text { positive }\end{array}$ & $20(87 \%)$ & $72(96 \%)$ \\
\hline $\begin{array}{c}\text { Lowenstein } \\
\text { Jenson (solid) } \\
\text { positive }\end{array}$ & $17(74 \%)$ & $3(4 \%)$ \\
\hline
\end{tabular}

Table 3: Rates of recovery of Mycobacteria by the BACTEC MIGIT 960 liquid system and Lowenstein Jenson solid medium in 98 out of the 943 study participants' documents with suggestive chest $x$-ray findings. 
The total number of organisms recovered was $98(23+75)$ of which 23 were M. tuberculosis while 75 were non-tuberculosis mycobacterium (NTM). Chi-square test for differences in recovery of M. tuberculosis BACTEC MGIT 960 versus LJ medium, $p>0.05$ was not significant. In this study significant difference was not demonstrated statically between BACTEC MIGIT 960 and LJ medium in terms of Mycobacterial recovery rate $(p>0.05)$ (Table 3$)$.

\subsection{Drug Susceptibility Pattern of M. Tuberculosis}

The BACTEC MGIT 960 susceptibility testing for Streptomycin (S), Isoniazid (I), Rifampin (R) and Ethambutol (E), called SIRE, is a rapid and qualitative procedure for establishing susceptibility of $M$. tuberculosis for the four drugs using critical test concentrations. The susceptibility of the 23 culture positive isolates was as shown below (Table 4).

\begin{tabular}{|c|c|c|c|c|}
\hline \multirow{2}{*}{$\begin{array}{c}\text { Name of the } \\
\text { Drug }\end{array}$} & \multicolumn{2}{|c|}{ Susceptible isolates } & \multicolumn{2}{c|}{$\begin{array}{c}\text { Resistant isolates } \\
\text { No }\end{array}$} \\
\cline { 2 - 5 } & Number & Percentage & Number Percentage \\
\hline Isoniazid & 16 & $70 \%$ & 7 & $31 \%$ \\
\hline Rifampcin & 21 & $91 \%$ & 2 & $8 \%$ \\
\hline Ethambutol & 17 & $74 \%$ & 6 & $26 \%$ \\
\hline Streptomycin & 8 & $78 \%$ & 5 & $22 \%$ \\
\hline MDR & 0 & 0 & 2 & $8 \%$ \\
\hline
\end{tabular}

Table 4: Number and percentage of $M$. tuberculosis isolates susceptible to first line anti- TB agents.

Based on table 4 above; the resistance patter to Isoniazid; Rifampin, Ethambutol hydrochlorid and streptomycin was $7(30.5 \%) ; 2(8 \%) ; 6(26 \%)$ and $5(22 \%)$ respectively.

2/943(2.1\%) participants' documents with abnormal chest radiographs were found to be MDR. i.e. 2/23 culture positive isolates. Eleven (11/23) isolates were found to be mono drug resistant (Resistant to one of isoniazid, rifampin, ethambutol hydrochloride or Streptomycin).

\section{Discussion}

Out of the total 943 study participants' documents with suggestive chest X-ray findings, 481(51\%) were male and 462(49\%) were female. This was in agreement with the study done by Kassu et al; But significantly different from the study done by Christian et al where $61 \%$ were males and 39\% were females [8,24]. Three hundred nine $(32.4 \%)$ of the study participants were in the age range of 29-44, which indicates that this age group are more likely to migrate for better job and opportunities abroad. Risk factors for pulmonary

\section{Virology \& Immunology Journal}

tuberculosis disease included middle age (29-44 years) as this group also constitutes the highest age group in the study. This is similar with the findings of Christian et al where $55 \%$ of these with X-ray suggestive of tuberculosis were in the age range of 25-44 years [11].

\section{Comparative Analysis of Chest X-Ray with Sputum Microscopy for Diagnosis of Tuberculosis}

Out of Nine hundred forty-three (943) samples with suggestive $\mathrm{X}$ - ray findings of tuberculosis, only $13(1.4 \%)$ were AFB positive. This means $98.6 \%$ were negative AFB smear cases of TB. In a study conducted on US bound immigrants and refugees $87 \%$ were smear negative AFB cases [7]. Their study further indicated $15.4 \%$ USA bound refugees in Africa were smear negative and inactive AFB. The prevalence in Somalia and Sudan in the study was $8.6 \%$ and $4.9 \%$ respectively. In a study conducted by Susan et al; pulmonary TB was diagnosed in $3.3 \%$ to $14 \%$ of immigrants and refugees classified overseas as having suspected active TB and $0.4 \%$ to $3.8 \%$ of those classified as having inactive tuberculosis [6]. Despite of its luck of specificity, chest X-ray is still widely used as stand-alone tool for diagnosis TB of the lung. However, practical experience and numerous studies have shown that no radiographic pattern is diagnostic of TB. Many diseases of the lung have a similar radiographic appearance that can easily mimic TB.

In our study, twenty-three $(\mathrm{n}=23)$ study participants' documents with suggestive chest X-ray findings were found to be positive Mycobacterial culture and identified as M. tuberculosis (PTB). Seventeen ( $n=17)$ whose smears were negative for AFB found to be positive for Mycobacterial culture and identified as M. tuberculosis (Table 1). This finding was in contrast to the study that was conducted in Swiss border where $22 \%$ of immigrants with chest x-ray abnormalities suggestive of pulmonary tuberculosis were documented by smear and /or culture [11]. According to Global Tuberculosis report 2010,163 new sputum smear positive cases of tuberculosis per 100,000 population and 378 all forms of TB new cases were reported in Ethiopia [5]. The report further indicated a prevalence 579 all forms of tuberculosis per 100,000 populations.

\section{Comparisons of the Sensitivity of Acid Fast Tuberculosis Microscopy with Culture}

Six $(0.6 \%)$ study participants' documents whose smear results were positive for AFB found to be positive for Mycobacterial culture and identified as $M$. 


\section{Virology \& Immunology Journal}

Tuberculosis. 17(1.8\%) visa applicants and refuges documents were negative for AFB smear but positive for Mycobacterial culture. 23 (2.4\%) had positive Mycobacterium tuberculosis culture results (pulmonary TB disease) and needed anti-tuberculosis treatment. Our findings are different from similar studies conducted elsewhere $[6,21,23]$.

\section{Antimicrobial Susceptibility Testing for $M$. Tuberculosis}

In our study, eleven $(11 / 23)$ isolates were found to be mono drug resistant (Resistant to one of isoniazid, rifampin, Ethambutol hydrochloride or Streptomycin whereas 2/943 (2.1\%) study participants' documents with abnormal chest radiographs were found to be MDR (2/23 culture positive isolates). The findings of our study are similar with the Ethiopian Ministry of Health report of TB 2010 which indicated $1.6 \%$ multidrug resistant cases among all new cases of infection [5]. Our study findings indicated that drug resistant to $M$. tuberculosis is significant public health concern and has to be addressed properly.

\section{Recommendations}

Based on the findings, the researcher makes the following recommendations;

- Training of laboratory technicians in AFB microscopy to increase the sputum processing and microscopy performance

- Strengthen laboratory quality assurance measures by promoting laboratory internal control measures, regular supervision and participating laboratories in external quality assessment scheme to monitor their diagnostic performance;

- Revision of the current tuberculosis diagnostic method in Ethiopia

- Capacity building to implement culture technique for diagnosis of tuberculosis at regional laboratory levels

The researchers also recommended further study to be conducted in the following.

- Prevalence of smear negative pulmonary TB

- Study on current TB drug resistance patterns in Ethiopia

\section{Conclusions}

Our study findings showed low detection rate of sputum microscopy and lack of specificity in chest X-ray. Therefore, there is a need to develop a scheme to determine the most cost-effective approaches for TB diagnosis method in the Ethiopian setting such as culture method and applying laboratory internal and external control schemes. Joint efforts are needed to make the culture method of TB diagnosis cost effective and applicable in resource limited countries like that of Ethiopia.

\section{References}

1. Bañuls AL, Sanou A, Anh NT, Godreuil S (2015) Mycobacterium tuberculosis: ecology and evolution of a human bacterium Journal of Medical Microbiology 64(11): 1261-1269.

2. Palomino JC, Leao SC, Ritacco V (2007) Tuberclosis 2007; from basic science to patient care pp: 687.

3. Parsons LM, Somoskövi A, Gutierrez C, Lee E, Paramasivan CN, et al. (2011) Laboratory Diagnosis of Tuberculosis in Resource-Poor Countries: Challenges and Opportunities. Clinical microbiology reviews 24(2): 314-350.

4. Steingart KR, Ng V, Henry M, Hopewell PC, Ramsay A, et al. (2006) Sputum processing methods to improve the sensitivity of smear microscopy for tuberculosis: a systematic review. Lancet Infect Dis 6(10): 664-674.

5. World Health Organization (2010) WHO report 2010 Global tuberculosis control surveillance, planning, financing, WHO/HTM/TB/2010.

6. Maloney SA, Fielding $\mathrm{KL}$, Laserson $\mathrm{KF}$, Jones $\mathrm{W}$, Nguyen TN, et al. (2006) Assessing the performance of overseas tuberculosis screening Programs. A study among US-bound immigrants in Vietnam. Arch Intern Med 166(2): 234-240.

7. Liu Y, Weinberg MS, Ortega LS, Painter JA, Maloney SA (2009) Overseas screening for tuberculosis in U.S.bound immigrants and refugees. $\mathrm{N}$ Engl J Med 360: 2406-2415.

8. Rageade F, Picot N, Blanc-Michaud A, Chatellier S, Mirande C, et al. (2014) Performance of solid and liquid culture media for the detection of Mycobacterium tuberculosis in clinical materials: meta-analysis of recent studies. Eur J Clin Microbiol Infect Dis 33(6): 867-870.

9. Salman H, Sabine R (2006) Mycobacterium Growth Indicator Tube (MGIT) procedure manual. Find Diagnostics pp: 1-52. 


\section{Virology \& Immunology Journal}

10. Lobue PA, Moser KS (2004) Screening of immigrants and refugees for pulmonary tuberculosis in San Diego County, California. Chest 126(6): 1777-1782.

11. Christian M, Yolande B, Pierre B, Jean-Pierre Z (2007) Active screening for pulmonary tuberculosis by chest x-ray among immigrants at the Swiss border. SWISS MED WKLY 137(45-46): 649-654.

12. Troper LE, Laserson KL, Cookson ST (2004) Infectious tuberculosis among newly arrived refugees in the United States. N Engl J Med 350: 2105-2106.

13. Ebrahimzadeh A, Mohammadifard M, Naseh G (2014) Comparison of Chest X-Ray Findings of Smear Positive and Smear Negative Patients with Pulmonary Tuberculosis. Iran J Radiol 11(4): e13575.

14. Zellweger JP, Heinzer R, Touray $M$, Vidondo $B$, Altpeter E (2006) Intra-observer and overall agreement in the radiological assessment of tuberculosis. Int J Tuberc Lung Dis 10(10): 11231126.

15. Behr MA, Warren SA, Salamon H, Hopewell PC, Ponce de Leon A, et al. (1999) Transmission of Mycobacterium tuberculosis from patients' smearnegative for acid-fast bacilli. Lancet 353(9151): 444449.

16. Hines N, Payeur JB, Hoffman LJ (2006) Comparison of the recovery of Mycobacterium bovis isolates using the BACTEC MGIT 960 system, BACTEC 460 system, and Middle brook 7H10 and 7H11 solid media. J Vet Diagn Invest 18(3): 243-250.

17. Apers L, Mutsvangwa J, Magwenzi J, Chigara N, Butterworth A, et al. (2003) A comparison of direct microscopy, the concentration method and the Mycobacteria Growth Indicator tube for the examination of sputum for acid-fast bacilli. Int J Tuberc Lung Dis 7(4): 376-381.

18. Lu D, Heeren B, Dunne WM (2002) Comparison of the automated Mycobacteria growth indicator tube system (BACTEC 960/MGIT) with Löwenstein-Jensen medium for recovery of Mycobacteria from clinical specimens. Am J Clin Pathol 118(4): 542-545.

19. Hirooka T, Higuchi T, Tanaka N, Ogura T (2004) The value of proper sputum collection instruction in detection of acid-fast bacillus. Kekkaku 79(2): 33-37.

20. Gupta R, Espinal MA, Raviglion MC (2004) Tuberculosis as a major global health problem in the 21st century: a WHO perspective. Semin Respir Crit Care Med 25(3): 245-253.

21. Rodrigues C, Shenai S, Sadani M, Sukhadia N, Jani M, et al. (2009) Evaluation of the BACTEC MIGIT 960 TB system for recovery and identification of mycobacterium tuberculosis complex in a high through put tertiary care centre. Indian Journal of Medical Microbiology 27(3): 217-221.

22. Phillips PPJ, Mendel CM, Nunn AJ, McHugh TD, Crook AM, et al. (2017) A comparison of liquid and solid culture for determining relapse and durable cure in phase III TB trials for new regimens. BMC Med 15(1): 207.

23. Vincent V, Brown-Elliot B, Jost KC, Wallace RJ (2003) Mycobacterium: Phenotypic and genotypic identification. (8th edn), ASM Press: Washington DC.

24. Desta K, Asrat D, Lemma E, Gebeyehu M, Feleke B (2009) Prevalence of smear negative pulmonary tuberculosis among patients visiting St. Peter tuberculosis specialized hospital, Addis Ababa, Ethiopia. Ethiopia Med J 47(1): 17-24.

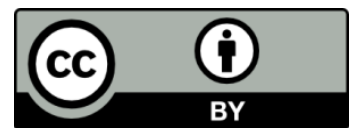

